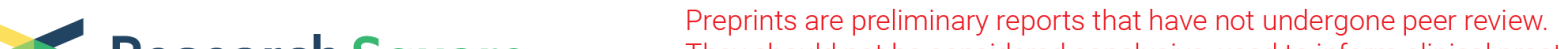 Research Square They should not be considered conclusive, used to inform clinical practice, or referenced by the media as validated information.
}

\section{A new efficient and comprehensive asthenopia questionnaire}

\section{Ruzhi Deng}

School of Optometry and Ophthalmology and Eye Hospital of Wenzhou Medical University

\section{Yanyan Lin}

School of Optometry and Ophthalmology and Eye Hospital of Wenzhou Medical University

\section{Jiafan Zhang}

School of Optometry and Ophthalmology and Eye Hospital of Wenzhou Medical University

\section{Yu Zhu}

School of Optometry and Ophthalmology and Eye Hospital of Wenzhou Medical University Jingwei Zheng

School of Optometry and Ophthalmology and Eye Hospital of Wenzhou Medical University Xiaoman Li

School of Optometry and Ophthalmology and Eye Hospital of Wenzhou Medical University $\mathrm{Na}$ Lin

School of Optometry and Ophthalmology and Eye Hospital of Wenzhou Medical University Frank Thorn

School of Optometry and Ophthalmology and Eye Hospital of Wenzhou Medical University Fan Lu ( $\square$ lufan62@mail.eye.ac.cn )

School of Optometry and Ophthalmology and Eye Hospital of Wenzhou Medical University

\section{Research Article}

Keywords: Asthenopia, Reliability, Validity, Questionnaire, Delphi Method

Posted Date: January 9th, 2019

DOI: https://doi.org/10.21203/rs.2.190/v1

License: (c) (1) This work is licensed under a Creative Commons Attribution 4.0 International License. Read Full License 


\section{Abstract}

Background: There has been no standardized questionnaire and objective criterion for assessing and diagnosing asthenopia so far. This study aimed to design a valid and reliable questionnaire to determine the various causes of asthenopia for use by clinicians and researchers.

Methods: We developed a 19-item asthenopia questionnaire (AQ-19) in two phases. In the Design phase, 17 experts, 98 patients with asthenopia and 20 controls participated to generate the item list. We generated a comprehensive 52 -item list to create our 1 st version. The list included selected items based on a literature review, patient interviews and the Delphi experts method. In the Validation phase, we conducted a pilot test (127 patients and 25 controls) and retest (154 patients and 24 controls) for item reduction and a questionnaire validity assessment.

Results: Our initial questionnaire contained 52 symptoms and 2 self-evaluation questions. After item reduction and assessment, we generated a 19-item questionnaire. It included 19 symptom items that were classified into three domains through factor analysis. Cronbach's a for the three subscales of this version were between 0.79 and 0.85 , while for the complete questionnaire Cronbach's a was 0.90 , with a split-half reliability of 0.80 . Factor analysis showed the three components have eigenvalues $>3$ and these explained $54.3 \%$ of the variance.

Conclusions: The AQ-19 has acceptable psychometric properties, making it a valid and reliable tool for ophthalmologists and optometrists to evaluate asthenopia as well as to provide clues to find its causes. It provides three levels of information: (1) Identifies the presence of asthenopia. (2) Differentiates three major causal classifications (ocular-surface, systemic, and vision symptoms). (3) Focuses in on a specific cause. The clinician must still use his or her skills to determine the exact cause. The AQ-19 questionnaire has the potential to be used in clinical trials and outcome research, and most importantly to help clinicians efficiently and effectively diagnose the cause of a patient's asthenopia.

Trial registration: KYK [2016]8. Registered at 25 February 2016. Retrospectively registered.

Keywords: Asthenopia, Reliability, Validity, Questionnaire, Delphi Method

\section{Introduction}

The prevalence of asthenopia is increasing today. This has been reported to be as a result of changes in lifestyle and the wide use of computers, smartphones, e-book readers and video games [1]. A recent study of US students showed that $65 \%$ experienced digital eye strain, and adults under age 30 years experienced higher rates of digital eye strain symptoms (73\%) compared with other age groups [2]. The prevalence of asthenopia in Australian, Indian and Brazilian teenagers varied from $12.6 \%$ to $32.0 \%$ [3]. For Chinese college students, it was reported to be $65 \%$ in Beijing's Haidian district [4], 57\% in Xi'an [5], and $17 \%$ in Shanghai [6]. These studies indicate that asthenopia has become a common health problem 
worldwide. But the ways to identify asthenopia are so varied and in consistent that we cannot compare these percentages in a meaningful way.

Asthenopia, also called visual fatigue, is considered as a syndrome that includes visual discomfort and ocular-related symptoms such as headache, watery, burning or itching eyes, blurred vision, eye ache, dry eye sensation and diplopia. Ocular causes include uncorrected ametropia, binocular vision dysfunction, dry eye and screen fatigue, while systemic causes include anxiety, depression, and insomnia.

The patient complaint of asthenopia is a vexing problem for clinicians around the world. Many ophthalmologists do not focus on refractive error, binocular vision, or presbyopia; many optometrists lack training in eye disease. Thus, each group has difficulty assessing the full range of asthenopia causes. A broad asthenopia questionnaire can provide a valid starting point for all eye doctors to understand their patients' problem. In this way, they give patients specific suggestions for treating the symptoms and root causes.

Usually, ophthalmologists and optometrists diagnose two aspects of asthenopia. One is the objective signs, and the other is the subjective complaints of patients. However, so far, there has been no standardized questionnaire and objective criterion for assessing and diagnosing asthenopia.

Current questionnaires that attempt to assess visual fatigue caused by different factors such as VDT (visual display terminal) [7-10], dry eye [11-20], or binocular vision dysfunction[21-30] are one-dimensional and do not assess asthenopia comprehensively. Such questionnaires were used to assess asthenopia in previous studies. In 2008, Kuze and Ukai produced a 28 -item questionnaire using a 7-point scale for a list of 5 types of visual fatigue symptoms [31]. Heuer et al. assessed visual fatigue using a 10-point scale, six-item questionnaire to assess visual fatigue [32]. Ogata et al. [33] and Amalia [34] presented a series of visual fatigue symptoms in their questionnaire. However, these questionnaires did not classify asthenopia qualitatively or quantitatively. Thus, we attempted to design a more comprehensive questionnaire that included the appropriate symptoms covering all aspects of visual fatigue to help ophthalmologists and optometrists evaluate patients' subjective feelings and provide the clues to find the causes of asthenopia.

We used complex statistical procedures to provide clinicians with an effective, efficient tool to understand a patient's problem at 3 levels:

1. To identify a patient's complaint as asthenopia. This is relatively simple.

2. To differentiate the three general classes of clinical problems causing the asthenopia. Ordinarily this may take a clinician considerable time and effort trying to interpret a patient's ad lib comments.

3. Focus in more closely on the specific cause of the problem. The precise identity of the problem can only be approximated within the realm of the questions asked in the questionnaire. This quickly brings the clinician close to the exact patient problem. It cannot, for example, differentiate between a corneal scratch, a corneal infection, or a corneal lesion. The clinician must still use his or her skills to determine the exact cause. 


\section{Methods}

Creating an efficient, effective questionnaire is a time-consuming task involving literature reviews, expert opinions, patient feedback, and complex statistical analyses to find the set of questions and response choices that identify your target information (effectivity) and require the least amount of time and confusion by the patient (efficiency). In the procedures we describe the literature search that found most of the questions experts asked patients to detect and identify asthenopia. Duplicates were eliminated and a committee of experts were asked to omit questions they thought were not useful, thereby shortening the questionnaire. The 45 remaining questions were administered to 127 asthenopia patients and 25 control patients. The factor analysis was applied to identify questions that did not correlate with the most effective questions or that almost duplicated them. People administering the questions also identified questions the patients did not understand, found frustrating, or took a long time answering. Most of these were eliminated. The remaining 19 questions could be answered on the questionnaire quickly with minimal problems. This short questionnaire was then tested for statistical efficiency on a large sample (152 asthenopia patients and 24 controls) for the efficiency needed to be identified as a statistically efficient, effective questionnaire. The clinicians administering it also thought it was both efficient and effective as a clinical tool. The process used to describe how the questionnaire was developed and proved its use in identifying asthenopic patients is described in detail in this article.

\section{Subjects}

We performed this study between June 2015 and June 2016 at the Wenzhou Medical University Affiliated Eye Hospital. The study was approved by the ethics committee of the Eye Hospital of the Wenzhou Medical University and adhered to the tenets of the Declaration of Helsinki. The patients from the Eye Hospital were enrolled after written informed consent. Inclusion criteria were: (1) complaints of eye fatigue and (2) a clear diagnosis of asthenopia. The exclusion criteria were: cannot read or understand the questionnaire.

In the three rounds of patient interviews, patients were interviewed on the day they arrived at the hospital. We recorded the questions that were not fully understood for later revision of the text.

\section{Procedures}

The items to be included in the first version of the questionnaire were selected from a literature review. Then the items were further assessed by patient interviews and the Delphi method to form the 2nd version of the questionnaire. The questionnaire was then validated for reliability and validity analysis by a pilot test and retest (Figure 1). All procedures were performed in accordance with the tenets of the Declaration of Helsinki.

First phase (design)

Step 1 Literature review 
We applied two search strategies. "Asthenopia” was the most commonly used term but other terms were also used, such as visual fatigue, visual comfort and visual symptom. The first strategy was to search PubMed using these different terms for asthenopia, and questionnaire, scale, question, interview. The second strategy was based on the most common etiology of asthenopia and included visual display terminal, computer vision syndrome, dry eye, binocular vision, convergence insufficiency (Cl), accommodation insufficiency (AI), and questionnaire, scale, question, interview.

\section{Step 2 Patient interviews}

We selected items for the first questionnaire based on the results of the literature review. We then interviewed 98 asthenopia patients and 20 symptom-free controls to compare responses for each item in the first questionnaire. We then calculated the response rate. If an item was mentioned by less than $20 \%$ of the patients, it was deemed that it did not represent a widely-held concern.

Step 3 Delphi method

We then conducted a Delphi method using insights from expert clinicians to modify the first questionnaire. The Delphi method is a multistage survey involving rounds of structured questions delivered by a panel of identified experts[35]. Its objective is to obtain the most reliable consensus using intensive questionnaires that provided feedback as "controlled opinion".

We analyzed the first version of the questionnaire through a three-round Delphi consultation (Figure 2).

Most studies use panels of 15 to 35 people. We recruited 17 experts who met the following criteria: (1) clinical familiarity with asthenopia; (2) qualification in optometry or ophthalmology; (3) relevant clinical experience exceeding 10 years; (4) Postgraduate clinical education.

After expert panel members were selected, three rounds of Delphi comparisons were conducted. In the first round, we asked experts to mark each item of the 1st questionnaire according to its importance. During this round, experts could raise questions about items and propose new items.

In each Delphi method round, we analyzed the mean and coefficient of variation of the scores for each item to determine the consensus of the experts' opinions.

In the second Delphi method round, we returned feedback from the results of the first round of questionnaires to each expert and to the consultants who provided the questionnaires. Feedback content contained the mean score, coefficient of variation and the scores they made in the last round. The expert was permitted to change his or her mind in the second round and re-score the items of the questionnaire.

The Delphi method was repeated until the value of Kendall's coefficient reached a standard. Kendall's coefficient of concordance $(\mathrm{W})$ was calculated to determine the degree of consensus among the experts. In Delphi studies, it is common to conduct additional rounds to see if consensus can be improved.

Second phase (validation) 
There is no gold standard for item reduction at this time. Rather, a group of statistical assessments is used to evaluate the responses to the questionnaire. All of the methods and item reduction criteria have been described previously[36]. We interviewed 127 asthenopia patients and 25 controls to do the item reduction. This enabled us to develop the 3rd version of the questionnaire.

We assessed each item according to 7 principles: (1) item-total correlation; (2) correlation with the scores of self-evaluation questions (questions 1 and 2); (3) item-total correlation after correction; (4) alteration in Cronbach's $\otimes$ after item reduction; (5) factor loadings; (6) differences between patients and controls, and (7) response rate. If the item met the reduction principle, then we scored 1 point. If the item had more than 4 points, it was reduced. Details are shown in Table 1.

\section{Step 5 Retest}

Considering that many items were reduced in the previous steps, we interviewed an additional 152 asthenopia patients and 24 controls to review the validation and reliability of the questionnaire. We applied a similar method to that used for the pilot test. Based on this review, we finalized the 4th version of the questionnaire to differentiate the causes of asthenopia.

Statistical analysis

Statistical analyses were conducted using SPSS19.0. Exploratory factor analysis was performed to reduce items and derive the subscale that each item belongs to. Meaningful loadings were assessed using the criteria of 0.32 (poor), 0.45 (fair), 0.55 (good), 0.63 (very good), or 0.71 (excellent) [37]. Internal consistency was calculated for all resulting subscales, using Cronbach's a coefficient. All tests were twotailed, with a $p$ value $<0.05$ considered significant.

\section{Results}

First phase (design)

Step 1 Literature review

Our search selected 29 relevant questionnaires: 5 asthenopia questionnaires, 10 dry eye questionnaires, 10 binocular vision questionnaires and 4 VDT questionnaires. These are shown in Table 2 7-34.

We selected 52 symptoms based on the results of the literature review and measured the frequency of occurrence and intensity of each symptom. Frequency was scored on a scale of 0-3 points: never $=0$, occasionally $=1$, often $=2$, and always $=3$. Four levels of symptom strength or intensity were graded similarly, where mild $=1$, moderate $=2$ and intense $=3$. Symptom frequency scored as never occurring was 
rated as 0 (none) on the intensity scale. We used the following expression to calculate a total score on the questionnaire:

\section{Score}

This expression incorporates both the frequency and intensity of symptoms, given that both are clinically important.

It is uncertain which is more important in establishing the clinical significance of a condition: whether a particular symptom is occasional but intense or frequent but mild.

Lacking a gold standard instrument for assessing asthenopia, we added two self-evaluation questions to assess the degree of asthenopia according to its definition. These were:

Q1: "Have you felt eye strain in the past two weeks?"

Q2: "Have the symptoms of eye strain influenced your study, work or daily life?"

We assumed that the total score of the two self-evaluation questions reflected the severity of asthenopia.

The 1st questionnaire contained 2 self-evaluation questions and 52 items related to ocular-surface symptoms, visual quality, close-work-related ocular symptoms, systemic symptoms and driving-related symptoms.

\section{Step 2 Patient interviews}

A total of 98 subjects participated in the patient interview, consisting of $47(48.4 \%)$ males and $50(51.6 \%)$ females. Mean age of the subjects ( \pm SD) was $34.4 \pm 14.6$ years.

Step 3 Delphi method

Agreement rates for the three rounds of the Delphi method were $100 \%$, indicating high concordance within our expert panel. The expert authority coefficient $(\mathrm{Cr})$ was more than 0.6 , which is considered to be good.

The statistical results of the first round of the Delphi method and patient interviews are shown in Table 3.

The Delphi method resulted in a Kendall's W of 0.40, which represents a moderate level of consensus among panelists (Schmidt, 1997)[38]. The Kendall's coefficients of the three rounds of the Delphi method are as shown in Table 4.

Following application of the Delphi method, we deleted, merged and added questions from the earlier questionnaire and then developed the 2 nd version of the questionnaire. 
We reduced 9 items, such as 'Feeling of friction around the eyes' and 'Colored halos around objects'. We merged two or three similar items, for example, Q22, Q25 and Q43 questions related to losing sight, into: 'Does your sight fluctuate during a single day'. We amended some items by adding conditions, for example for Q48 and Q49, where we added the following: "Have you felt depressed because of your eye symptoms?" to assess any influence from ocular symptoms. After optimizing the 1st questionnaire, we developed the 2 nd questionnaire that included 2 self-evaluation questions and 42 items related to ocularsurface symptoms, visual quality, close-work-related symptoms, systemic symptoms and driving-related symptoms.

Second Phase: (validation)

Step 4 Pilot test

A total of 127 subjects participated in the pilot test, with 124 valid data, consisting of $47(37.9 \%)$ males and $77(62.1 \%)$ females. Mean age of the subjects $( \pm S D)$ was $31.1 \pm 10.9$ years.

After item reduction, Q2, Q3, Q4, Q5, Q6, Q14, Q15, Q16, Q18, Q19, Q22, Q24, Q26, Q28, Q32, Q35, Q39 were chosen to be omitted. However, we considered that Q20 and Q34 were a priori important and indispensable. Thus, we chose to retain these two items. In this round, 16 items were omitted and 28 items were retained.

For the evaluation of construct validity, we conducted a factor analysis for the 3rd version with varimax rotation. Our scree plot (Figure 3) showed an inflexion that justified retaining three components. The factor loadings after rotation are shown in Table 5. Items that clustered in the same factors were (1) ocular-surface symptoms, (2) vision symptoms, and (3) systemic symptoms. For the 3rd version, three of the factors had eigenvalues $>3$. Together these factors explain $47.87 \%$ of the total variance. Cronbach's a for the three subscales of the 26 -item version were between 0.76 and 0.88 (Table 5).

\section{Step 5 Retest}

A total of 152 subjects participated in the retest, with valid data from 151 . There were $50(33.1 \%)$ males and $101(66.9 \%)$ females and the mean age was ( \pm SD) $37.3 \pm 12.9$ years.

The scree plot (Figure 4) showed an inflexion that justified retaining three factors. The factor loadings after rotation are shown in Table 6. Items that cluster on the same factors suggest components 1 (ocularsurface symptoms), 2 (systemic symptoms), and 3 (vision symptoms). After item reduction, we omitted 7 items, namely Q5, Q7, Q15, Q16, Q17, Q18, Q19. For the 4th questionnaire, three components had eigenvalues $>3$. Together these components explain $54.34 \%$ of the variance. Cronbach's a for the three subscales of the 19-item version were between 0.79 and 0.85 (Table 6).

\section{Discussion}


Compared with previous asthenopia questionnaires, AQ-19 is the first comprehensive asthenopia questionnaire that quantitatively evaluates subjective feelings in both frequency and intensity. The responses to the two rating scales for each symptom are combined multiplicatively into one rating scale for the analysis, resulting in a single symptom severity score. AQ-19 included 2 self-evaluation questions and three aspects of asthenopia which include 7 ocular-surface symptoms, 7 vision symptoms and 5 systemic symptoms. The questionnaire was developed with wide consensus among the experts and researchers in the field and was well accepted by the target group, our patients. And it aims to give ophthalmologists and optometrists direction for discovering the causes of asthenopia.

The results obtained show that the AQ-19 has acceptable psychometric properties for asthenopia patients. Cronbach's a for the three subscales of this version were between 0.79 and 0.85 , while for the complete questionnaire Cronbach's a was 0.90 , with a split-half reliability of 0.80 . These values represent very good to excellent statistical power. Principal component analysis showed the three components have eigenvalues $>3$ and these explained $54.3 \%$ of the variance.

We believe that our questionnaire overcame the deficiencies in previously published versions. AQ-19 is was designed initially on the basis of complicated symptoms studied by other authors, as shown in our literature review. But it was given a refined focus that provides a comprehensive evaluation of the presence of symptoms, their frequency and perceived intensity, as well as being easily administered.

The AQ-19 is designed to be helpful to ophthalmologists and optometrists in identifying the causes of asthenopia. First, it ascertains in writing the presence of asthenopia. Next, it is divided into three parts and the scores for each part give examiners a guide as to whether the patient complaint is due to a physical problem such as dry eye or conjunctivitis or to a functional vision problem such as refractive error, binocular integration or VTD use or to a more systemic problem such as anxiety or depression. This guides the clinician into one of the three major causal areas and the individual questions provide the most common specific complaints for a possible diagnosis. And finally, it tells the clinician something about the severity and frequency of the complaint.

The results from our asthenopia patients appear to be valid and very reliable. Larger samples might provide a more definitive instrument but we think this should be done in multiple settings. Considering that there has been no standardized questionnaire nor objective criterion to provide a gold standard for assessing and diagnosing asthenopia, criterion validity cannot be statistically tested. We have simply defined asthenopia as "the patient says he or she is suffering from eye strain." This may seem like a very shallow definition, but it is the most practical definition for the practicing doctor. The purpose of the questionnaire is to help the clinician who must work with a patient who presents with eye strain (or eye ache or eye fatigue). The patient presents the complaint in his or her own words which may be confusing and imprecise. In our questionnaire, they say they have eye strain. Thus, our definition is perfectly suited to simplifying the patient visit. The repeatability of the questionnaire is difficult to test because asthenopia is not a constant condition in that it varies from day to day. And of course, the questionnaire requires patients to make subjective judgments, requiring comprehension and expression of their 
considered opinions. Because of this, children, the poorly educated and the mentally infirm may require assistance in answering the items in the questionnaire.

One of the most vexing and often time-consuming patient complaints in ophthalmology is asthenopia because it is so difficult to elicit the information needed to understand and diagnose a patient's problem. We have developed through a series of proven iterative procedures a questionnaire that is relatively short and convenient for the patient yet provides a great deal of information for the clinician. It clearly identifies the complaint as asthenopia, differentiates among the main classes of asthenopia (ocular-surface, systemic, and vision symptoms), and then provides the specifics of the asthenopia complaint as well as its intensity and frequency so the clinician can extract this information at a glance. This guides the clinician to do the appropriate clinical procedures to identify the exact cause of the asthenopia.

We expect the questionnaire to make a serious contribution to the practice of our profession and the wellbeing of our patients. It is being used in our system's three hospitals and several other neighboring hospitals and the feedback from clinicians has been supportive. To better serve the goal of the questionnaire in other cultures, we must test the instrument and provide data about its usefulness to make this a universally acceptable standard for the diagnosis of asthenopia. We expect to work with several clinics abroad to refine the instrument internationally. The questionnaire is provided in the supplement in digital form along with an application to provide the analysis for each patient.

\section{Abbreviations}

\begin{tabular}{|c|c|c|c|}
\hline Abbreviation & Full name & Abbreviation & Full name \\
\hline AQ-19 & asthenopia questionnaire-19 & VDT & visual display terminal \\
\hline $\mathrm{Cl}$ & convergence insufficiency & $\mathrm{Al}$ & accommodation insufficiency \\
\hline $\mathrm{Cr}$ & expert authority coefficient & CANDEES & $\begin{array}{l}\text { The Canada Dry Eye Epidemiology } \\
\text { Study }\end{array}$ \\
\hline IDEEL & $\begin{array}{l}\text { Impact of Dry Eye on } \\
\text { Everyday Life }\end{array}$ & OSDI & Ocular Surface Disease Index \\
\hline DEEP & $\begin{array}{l}\text { Dry Eye Epidemiology } \\
\text { Projects }\end{array}$ & CIRS & $\begin{array}{l}\text { Convergence Insufficiency and } \\
\text { Reading Study }\end{array}$ \\
\hline CISS & $\begin{array}{l}\text { Convergence Insufficiency } \\
\text { Symptom Survey }\end{array}$ & COVD-QOL & $\begin{array}{l}\text { College of Optometrists in Vision } \\
\text { Development Quality of Life } \\
\text { questionnaire }\end{array}$ \\
\hline NEI-VFQ & $\begin{array}{l}\text { National Eye Institute Visual } \\
\text { Function Questionnaire }\end{array}$ & VFQ-UI & $\begin{array}{l}\text { Visual Function Questionnaire-Utility } \\
\text { Index }\end{array}$ \\
\hline cVss & $\begin{array}{l}\text { Computer-Vision Symptom } \\
\text { Scale }\end{array}$ & CVS-Q & $\begin{array}{l}\text { Computer Vision Syndrome } \\
\text { Questionnaire }\end{array}$ \\
\hline
\end{tabular}

\section{Declarations}


The authors would like to thank all the study participants for making this study possible. We acknowledge Dr. Shunnan Yang for helping with the research design and Dr. Alan Johnston for his comments and English editing. The authors also would like to thank the experts who participated in the Delphi method and the colleagues from the Eye Hospital of the Wenzhou Medical University who helped with patient recruitment.

\section{Author contributions}

FL and RZD made substantial contributions to the conception and design of this study, and FL acted as the overall guarantor. JFZ and YYL conducted the data analysis, which were reviewed by JWZ and FT. YZ, $\mathrm{NL}$ and XML were responsible for patients recruitment and expert consulting rounds. YYL wrote the first draft of the manuscript text, and all authors provided feedback and revisions on the first and subsequent drafts. All authors approved the final submitted manuscript.

\section{Funding}

Supported by Wenzhou Municipal Science and Technology Bureau Grant $₫ 2016$ Y0648囚. The grants had no role in the design or conduct of this research.

Ethics Approval and Consent to participate

The study was approved by the ethics committee of the Eye Hospital of the Wenzhou Medical University and adhered to the tenets of the Declaration of Helsinki. The patients from the Eye Hospital were enrolled after written informed consent. All procedures performed in studies involving human participants were in accordance with the ethical standards of the ethics committee of the Eye Hospital of the Wenzhou Medical University and with the 1964 Helsinki declaration and its later amendments or comparable ethical standards. Informed consent was obtained from all individual participants included in the study.

Availability of Data and Materials

The datasets analyzed during the current study are available from the corresponding author on reasonable request.

Conflict of interest

The authors declare no competing financial interests.

\section{References}

1. Vilela MA, Castagno VD, Meucci RD, Fassa AG. Asthenopia in schoolchildren. Clinical ophthalmology. 2015;9:1595-603. doi:10.2147/OPTH.S84976. 
2. Eyes overexposed: the digital device dilemma [database on the Internet]. Consumer Electronics Show. 2016. Available from: https://www.thevisioncouncil.org/digital-eye-strain-report-2016.

3. Vilela MA, Pellanda LC, Fassa AG, Castagno VD. Prevalence of asthenopia in children: a systematic review with meta-analysis. Jornal de pediatria. 2015;91(4):320-5. doi:10.1016/j.jped.2014.10.008.

4. Ma L, Ahati A, Liu XT, Zou ZY, Li Y, Wang MF et al. [Epidemiology and related factor analysis of visual fatigue in university students of Haidian District of Beijing]. Beijing da xue xue bao Yi xue ban = Journal of Peking University Health sciences. 2011;43(3):365-9.

5. Han CC, Liu R, Liu RR, Zhu ZH, Yu RB, Ma L. Prevalence of asthenopia and its risk factors in Chinese college students. International journal of ophthalmology. 2013;6(5):718-22. doi:10.3980/j.issn.22223959.2013.05.31.

6. You XF, Tan H, Wang L. Epidemiology and related factor analysis of visual fatigue of primary and middle school students in Shanghai. Chinese Journal of School Health. 2015.

7. Mangione CM, Lee PP, Pitts J, Gutierrez P, Berry S, Hays RD. Psychometric properties of the National Eye Institute Visual Function Questionnaire (NEI-VFQ). NEI-VFQ Field Test Investigators. Archives of Ophthalmology. 1998;116(11):1496-504.

8. Rentz AM, Kowalski JW, Walt JG, Hays RD, Brazier JE, Yu R et al. Development of a preference-based index from the National Eye Institute Visual Function Questionnaire-25. Jama Ophthalmology. 2014;133(3):310-8.

9. Gonzalez-Perez M, Susi R, Antona B, Barrio A, Gonzalez E. The Computer-Vision Symptom Scale (CVSS17): development and initial validation. Investigative ophthalmology \& visual science. 2014;55(7):4504-11. doi:10.1167/iovs.13-13818.

10. Segui Mdel M, Cabrero-Garcia J, Crespo A, Verdu J, Ronda E. A reliable and valid questionnaire was developed to measure computer vision syndrome at the workplace. Journal of clinical epidemiology. 2015;68(6):662-73. doi:10.1016/j.jclinepi.2015.01.015.

11. Mcmonnies CW. Key questions in a dry eye history. Journal of the American Optometric Association. 1986;57(7):512-7.

12. Doughty MJ, Fonn D, Richter D, Simpson T, Caffery B, Gordon K. A patient questionnaire approach to estimating the prevalence of dry eye symptoms in patients presenting to optometric practices across Canada. Optometry \& Vision Science. 1997;74(8):624-31.

13. Rajagopalan K, Linda Abetz MA, Mertzanis P, Derek Espindle MA, MS CBO, Robin Chalmers OD et al. Comparing the Discriminative Validity of Two Generic and One Disease-Specific Health-Related Quality of Life Measures in a Sample of Patients with Dry Eye. Value in Health. 2005;8(2):168-74.

14. Schein OD, Tielsch JM, Muñoz B, Bandeen-Roche K, West S. Relation between Signs and Symptoms of Dry Eye in the Elderly: A Population-based Perspective. Ophthalmology. 1997;104(9):1395-401.

15. Magnan EM, Gittelson R, Bartels CM, Johnson HM, Pandhi N, Jacobs EA et al. Establishing chronic condition concordance and discordance with diabetes: a Delphi study. BMC family practice. 2015;16:42. doi:10.1186/s12875-015-0253-6. 
16. Schiffman RM, Christianson MD, Jacobsen G, Hirsch JD, Reis BL. Reliability and validity of the Ocular Surface Disease Index. Archives of Ophthalmology. 2000;118(5):615-21.

17. Oden NL, Lilienfeld DE, Lemp MA, Nelson JD, Ederer F. Sensitivity and specificity of a screening questionnaire for dry eye. Advances in experimental medicine and biology. 1998;438:807-20.

18. Schaumberg DA, Sullivan DA, Buring JE, Dana MR. Prevalence of dry eye syndrome among US women . American Journal of Ophthalmology. 2003;136(2):318-26.

19. Brown GC. Utility assessment among patients with dry eye disease. Ophthalmology. 2003;110(110):1412-9.

20. Bjerrum K. Dry eye symptoms in patients and normals. Acta Ophthalmologica Scandinavica. 2000;78(Suppl s231):14-5.

21. Borsting E, Rouse MW, De Land PN. Prospective comparison of convergence insufficiency and normal binocular children on CIRS symptom surveys. Convergence Insufficiency and Reading Study (CIRS) group. Optometry \& Vision Science Official Publication of the American Academy of Optometry. 1999;76(4):221-8.

22. Borsting EJ, Rouse MW, Mitchell GL, Scheiman M, Cotter SA, Cooper J et al. Validity and reliability of the revised convergence insufficiency symptom survey in children aged 9 to 18 years. Optometry \& Vision Science. 2003;80(12):832-8.

23. Grandolfo M, Vena GA, Angelini G, Bianchi B. Accommodation and the relationship to subjective symptoms with near work for young school children. Ophthalmic \& Physiological Optics the Journal of the British College of Ophthalmic Opticians. 2006;26(2):148-55.

24. Paul A. Efficacy of treatment for convergence insufficiency using vision therapy. Ophthalmic \& Physiological Optics. 2002;22(6):565-71.

25. Gallaway M, Scheiman M, Malhotra K. The effectiveness of pencil pushups treatment for convergence insufficiency: a pilot study. Optometry \& Vision Science Official Publication of the American Academy of Optometry. 2002;79(4):265-7.

26. Russell GE, Wick B. A prospective study of treatment of accommodative insufficiency. Optometry \& Vision Science Official Publication of the American Academy of Optometry. 1993;70(70):131-5.

27. Vaughn W, Maples WC, Hoenes R. The association between vision quality of life and academics as measured by the College of Optometrists in Vision Development Quality of Life questionnaire 1. Optometry (St Louis, Mo). 2006;77(3):116-23.

28. Borsting E, Chase C, Tosha C. Longitudinal study of visual discomfort symptoms in college students. Optometry \& Vision Science Official Publication of the American Academy of Optometry. 2008;85(10):992-8.

29. Rouse M, Borsting E, Mitchell GL, Kulp MT, Scheiman M, Amster D et al. Academic behaviors in children with convergence insufficiency with and without parent-reported ADHD. Optometry \& Vision Science Official Publication of the American Academy of Optometry. 2009;86(10):1169-77.

30. Birnbaum MH, Soden R, Cohen AH. Efficacy of vision therapy for convergence insufficiency in an adult male population. Journal of the American Optometric Association. 1999;70(4):225. 
31. Kuze J, Ukai K. Subjective evaluation of visual fatigue caused by motion images. Displays. 2008;29(2):159-66. doi:10.1016/j.displa.2007.09.007.

32. Heuer $H$, Hollendiek $G$, Kroger $H$, Romer $T$. [Rest position of the eyes and its effect on viewing distance and visual fatigue in computer display work]. Z Exp Angew Psychol. 1989;36(4):538-66.

33. Ogata M, Ukai K, Kawai T. Visual fatigue in congenital nystagmus caused by viewing images of color sequential projectors. Journal of Display Technology. 2005;1(2):314-20.

34. Amalia H, Suardana GG, Artini W. Accommodative insufficiency as cause of asthenopia in computerusing students. Universa Medicina. 2010;29(2).

35. Keil M, Tiwana A, Bush A. Reconciling user and project manager perceptions of IT project risk: a Delphi study 1. Information Systems Journal. 2002;12(2):103-19.

36. Tadic V, Cooper A, Cumberland P, Lewando-Hundt G, Rahi JS, Vision-related Quality of Life G. Development of the functional vision questionnaire for children and young people with visual impairment: the FVQ_CYP. Ophthalmology. 2013;120(12):2725-32. doi:10.1016/j.ophtha.2013.07.055.

37. Tosha C, Borsting E, Ridder WH, 3rd, Chase C. Accommodation response and visual discomfort. Ophthalmic \& physiological optics: the journal of the British College of Ophthalmic Opticians. 2009;29(6):625-33. doi:10.1111/j.1475-1313.2009.00687.x.

38. Schmidt K, Lou AM, Bruene D, Kenney M. Determining research priorities in pediatric nursing: A delphi study. Journal of Pediatric Nursing. 1997;12(4):201-7.

\section{Tables}

Table 1 The procedure used for item reduction

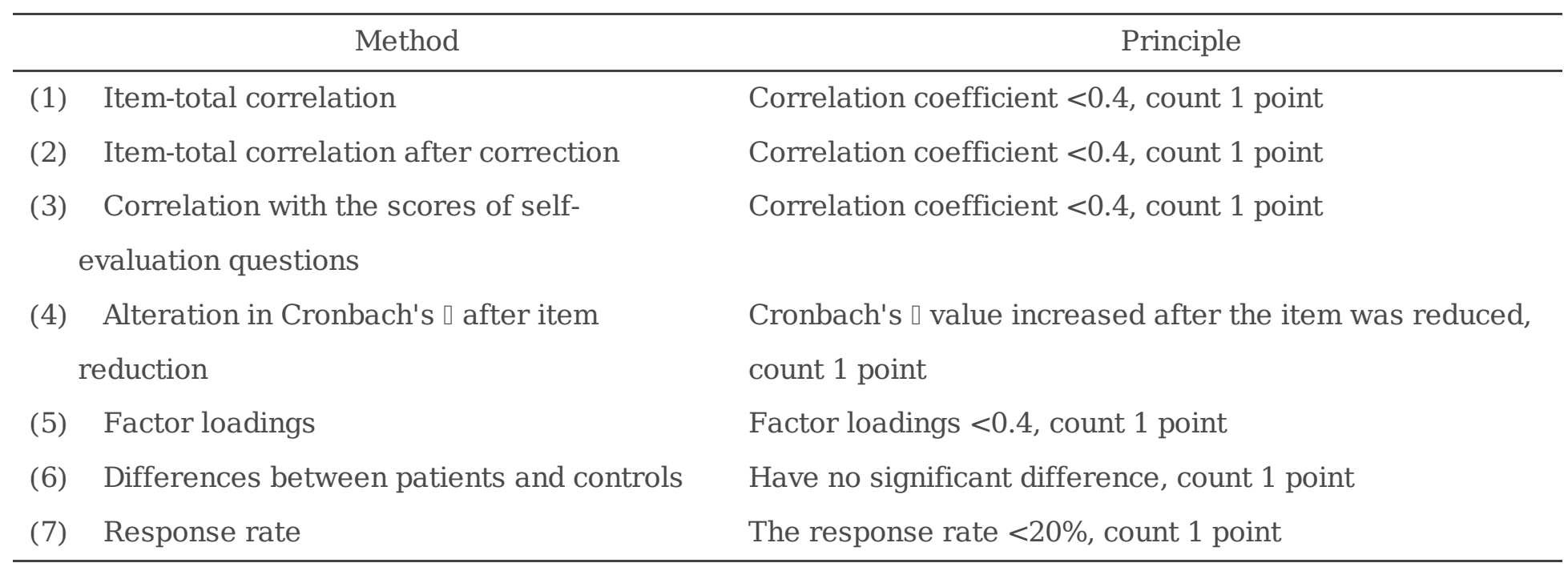

Table 2 Literature-sourced relevant questionnaires ${ }^{7-34}$ 


\begin{tabular}{cccc}
\hline VDT & Dry eye & Binocular vision & Asthenopia \\
\hline NEI-VFQ & McMonnies & CIRS & Kuze \\
\hline VFQ-UI & CANDEES & CISS16 & Heuer et al \\
\hline CVSS-17 & IDEEL & Sterner Questionnaire & Ogata \\
\hline CVS-Q & Salisbury Eye Evaluation & Adler Questionnaire & Amalia \\
\hline \multicolumn{5}{c}{ Dry Eye Questionnaire[DEQ } & Gallaway Questionnaire \\
\hline \multicolumn{5}{c}{ OSDI } & Rusell Questionnaire \\
\hline & DEEP & 19-Item COVD-QOL \\
\hline & Women's Health Study questionnaire & Conlon Survey \\
\hline & Utility assessment questionnaire & Academic Behavior Survey \\
\hline & Bjerrum questionnaire & Birnbaum Questionnaire \\
\hline
\end{tabular}

Legend : CANDEES: The Canada Dry Eye Epidemiology Study; IDEEL: Impact of Dry Eye on Everyday Life ;

OSDI: Ocular Surface Disease Index; DEEP: Dry Eye Epidemiology Projects; CIRS: Convergence Insufficiency and Reading Study;

CISS: Convergence Insufficiency Symptom Survey; COVD-QOL: College of Optometrists in Vision Development Quality of Life questionnaire;

NEI-VFQ: National Eye Institute Visual Function Questionnaire; VFQ-UI : Visual Function Questionnaire-Utility Index;

CVSS: Computer-Vision Symptom Scale; CVS-Q: Computer Vision Syndrome Questionnaire

Table 3 Kendall's coefficients for the three rounds of the Delphi method

\begin{tabular}{ccc}
\hline & the Kendall's coefficient & $P$ \\
\hline First round & 0.33 & $\square 0.001$ \\
Second round & 0.34 & $\square 0.001$ \\
Third round & 0.40 & $\square 0.001$ \\
\hline
\end{tabular}


Table 4 Rotated factor loadings $(\mathrm{n}=152)$ for the 4 th questionnaire

\begin{tabular}{|c|c|c|c|}
\hline \multirow[t]{2}{*}{ Items } & \multicolumn{3}{|c|}{ Factor } \\
\hline & 1 & 2 & 3 \\
\hline Q3 Eye pain & 0.77 & & \\
\hline Q8 Feeling of tightness around the yes & 0.71 & & \\
\hline Q4 Heavy eyelids & 0.63 & & \\
\hline Q6 Eye soreness & 0.62 & & \\
\hline Q2 Eye dryness & 0.61 & & \\
\hline Q1 Feeling uncomfortable around the eyes & 0.56 & & \\
\hline Q11 Increased sensitivity to light & 0.41 & & \\
\hline Q25 Sense of anxiety & & 0.81 & \\
\hline Q22 Have trouble keeping attention on reading & & 0.72 & \\
\hline Q23 Forgetful/poor memory when reading & & 0.72 & \\
\hline Q26 Sense of depression & & 0.70 & \\
\hline Q24 Dizziness or headache when reading & & 0.65 & \\
\hline Q12 Using much effort when doing near work & & & 0.71 \\
\hline Q13 Blurred vision & & & 0.71 \\
\hline Q14 Double vision & & & 0.68 \\
\hline Q10 Squinting your eyes & & & 0.64 \\
\hline Q20 Reading slowly & & & 0.63 \\
\hline Q9 Uncomfortable by the luminance of a screen & & & 0.61 \\
\hline Q21 Felt difficulty catching up with motion & & & 0.48 \\
\hline Eigenvalues & 3.48 & 3.44 & 3.41 \\
\hline$\%$ of variance & 18.31 & 18.12 & 17.92 \\
\hline Cronbach's $\alpha$ & 0.79 & 0.84 & 0.85 \\
\hline
\end{tabular}

Figures 
First phase

Design

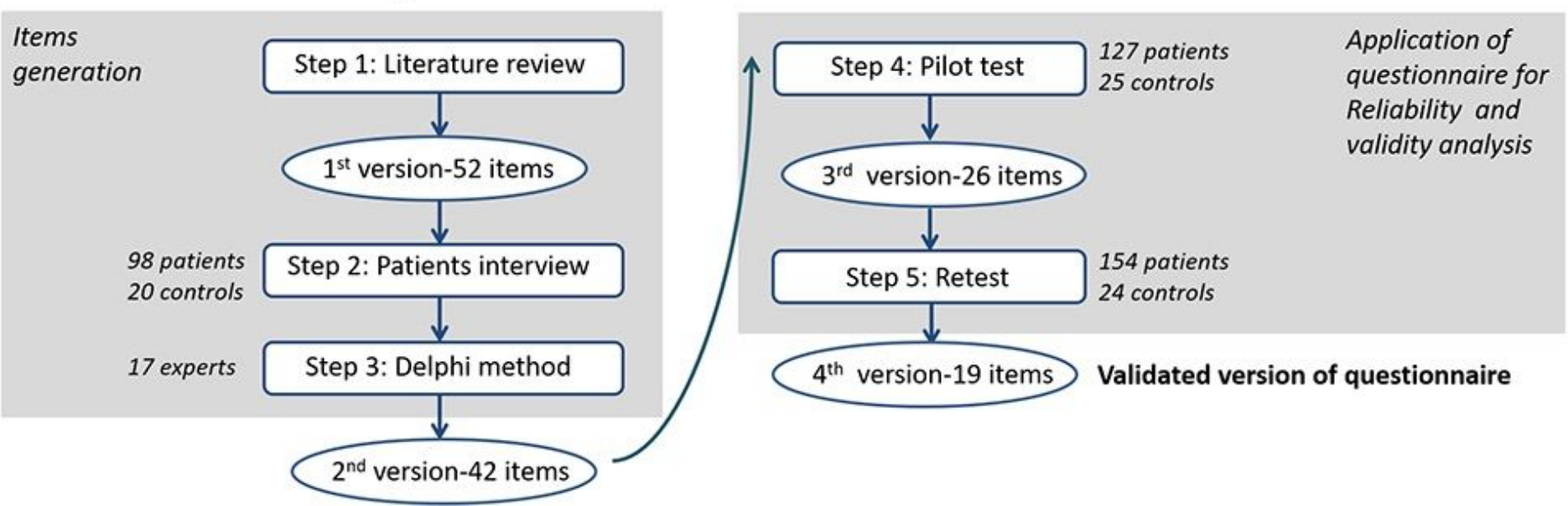

\section{Figure 1}

Steps in the design and subsequent validation of AQ-19. 


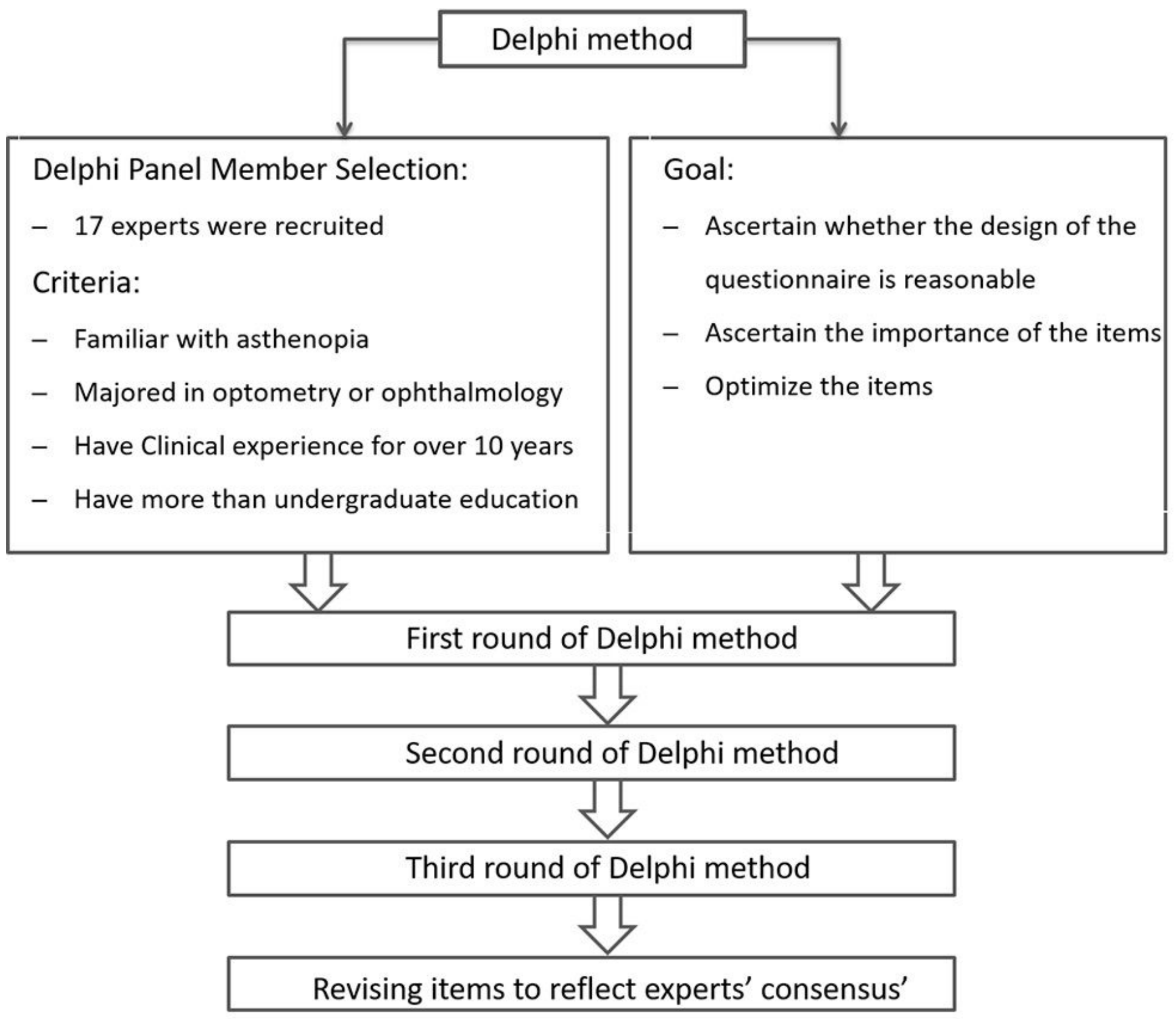

Figure 2

The Delphi method procedures. 


\section{Scree Plot}

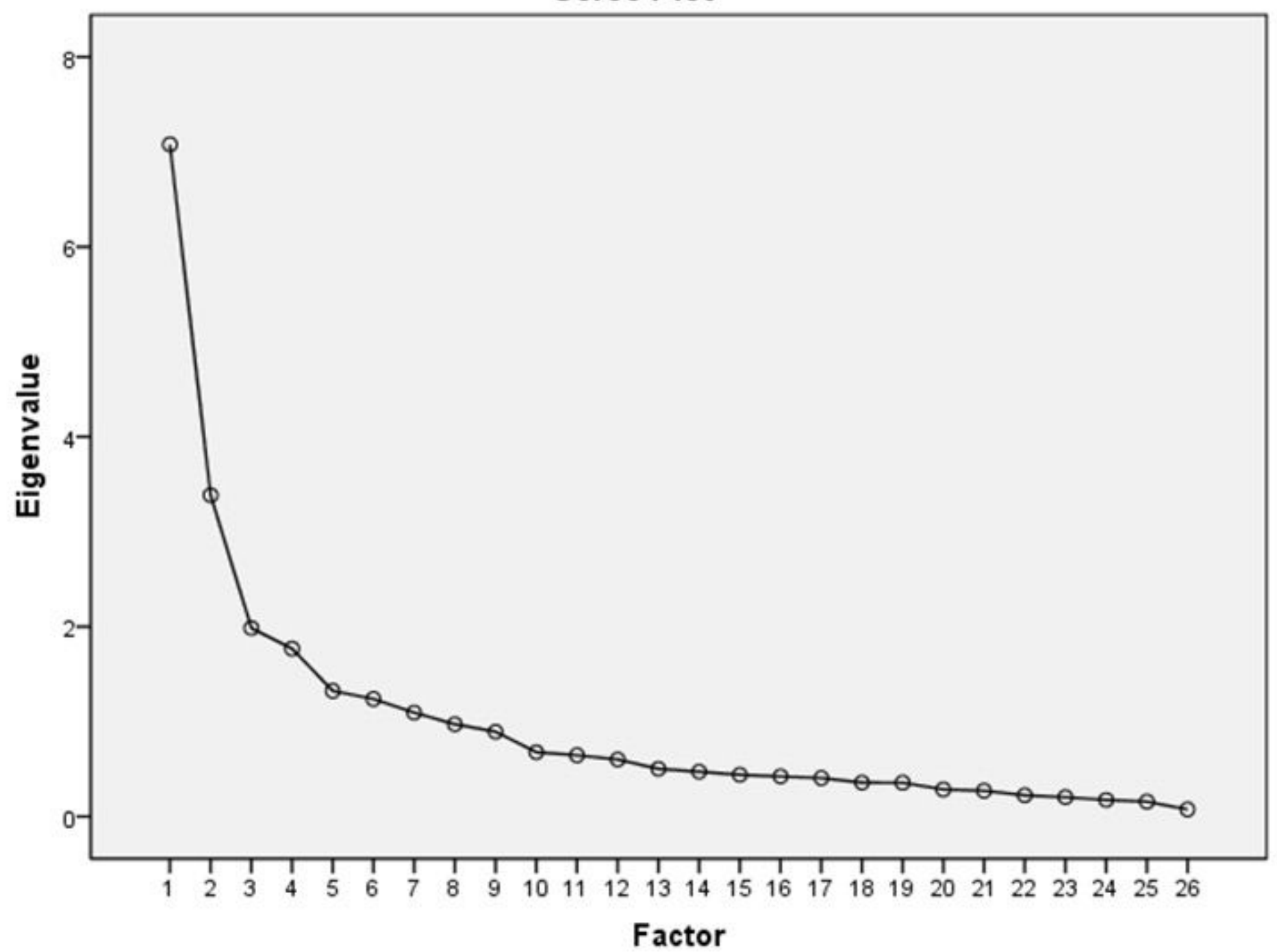

Figure 3

Scree plot for the 3rd questionnaire showing eigen values for main factor analysis. Three strong factors are evident on the steep phase of the curve. 


\section{Scree Plot}

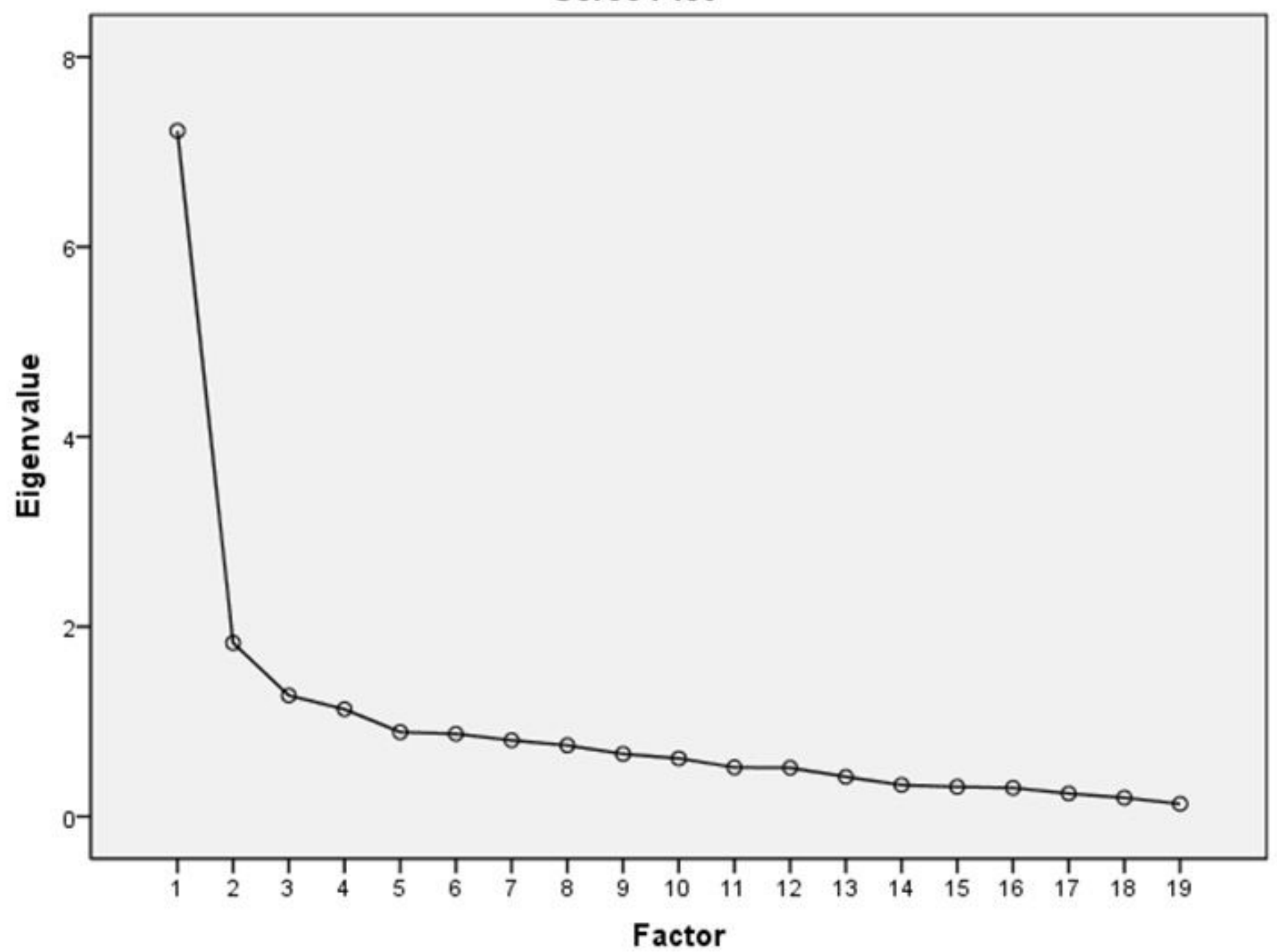

Figure 4

Scree plot for the 4th questionnaire showing eigen values for main factor analysis. Three strong factors are evident on the steep phase of the curve. 